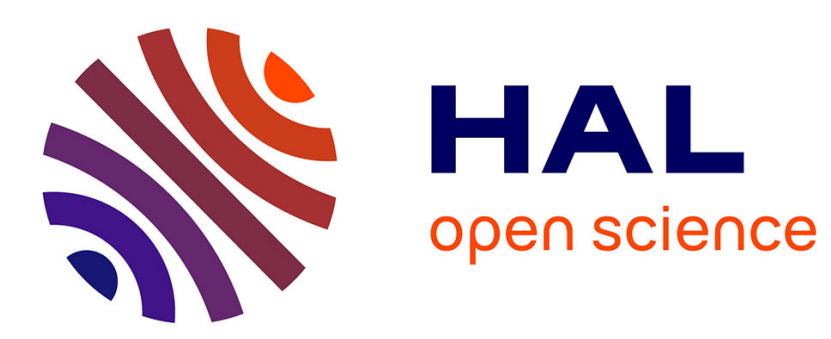

\title{
L2 Similarity Metrics for Diffusion Multi-Compartment Model Images Registration
}

Olivier Commowick, Renaud Hédouin, Emmanuel Caruyer, Christian Barillot

\section{To cite this version:}

Olivier Commowick, Renaud Hédouin, Emmanuel Caruyer, Christian Barillot. L2 Similarity Metrics for Diffusion Multi-Compartment Model Images Registration. 20th International Conference on Medical Image Computing and Computer Assisted Intervention - MICCAI, Sep 2017, Québec, Canada. pp.257-265, 10.1007/978-3-319-66182-7_30 . inserm-01556476

\section{HAL Id: inserm-01556476 https://www.hal.inserm.fr/inserm-01556476}

Submitted on 5 Jul 2017

HAL is a multi-disciplinary open access archive for the deposit and dissemination of scientific research documents, whether they are published or not. The documents may come from teaching and research institutions in France or abroad, or from public or private research centers.
L'archive ouverte pluridisciplinaire HAL, est destinée au dépôt et à la diffusion de documents scientifiques de niveau recherche, publiés ou non, émanant des établissements d'enseignement et de recherche français ou étrangers, des laboratoires publics ou privés. 


\title{
$\ell_{2}$ Similarity Metrics for Diffusion Multi-Compartment Model Images Registration
}

\author{
Olivier Commowick, Renaud Hédouin, Emmanuel Caruyer, Christian Barillot \\ VisAGeS U1228 INSERM/Inria, IRISA UMR CNRS 6074, Rennes, France
}

\begin{abstract}
Diffusion multi-compartment models (MCM) allow for a fine and comprehensive study of the white matter microstructure. Non linear registration of MCM images may provide valuable information on the brain e.g. through population comparison. State-of-the-art MCM registration however relies on pairing-based similarity measures where the one-to-one mapping of MCM compartments is required. This approach leads to non differentiabilties or discontinuities, which may turn into poorer registration. Moreover, these measures are often specific to one MCM compartment model. We propose two new MCM similarity measures based on the space of square integrable functions, applied to MCM characteristic functions. These measures are pairing-free and agnostic to compartment types. We derive their analytic expressions for multi-tensor models and propose a spherical approximation for more complex models. Evaluation is performed on synthetic deformations and inter-subject registration, demonstrating the robustness of the proposed measures.
\end{abstract}

\section{Introduction}

Diffusion weighted imaging (DWI) enables the in vivo study of the brain white matter (WM) microstructure. For this, the diffusion tensor (DT) has long been the model of choice for its simplicity and ability to provide quantitative scalar parameters (fractional anisotropy - FA, mean diffusivity - MD...) that correlate with pathology stages. However, the DT suffers from some limits when dealing with crossing fibers or since FA and MD encompass multiple microstructure properties that cannot be disentangled. In contrast, diffusion multi-compartment models (MCM) [10], i.e. a linear sum of diffusion compartments each modeling a fiber bundle, provide a more specific microstructure characterization.

Population studies enable the comprehension at the group level of a disease progression and severity. Typical population studies require the construction of atlases or the registration of all images to an atlas, on which the comparison is performed. For this task, using as much information as possible from the DWI, e.g. using the full MCM, enables a finer disease characterization [12]. With this objective, several methods have been explored for diffusion images registration.

Most approaches have focused on DT or DT-derived scalar images registration $[7,13]$. However, they do not use the whole diffusion information especially in crossing regions where the DT is limited. On the other end, some approaches [8] 
consider DWI registration. However, these algorithms are yet limited to singleshell DWI while the complete study of WM microstructure requires multiple b-values [11]. Moreover, adapted re-orientation in q-space is needed [6] and requires an underlying diffusion model making the registration model dependent.

MCM image registration has also been studied in [12] using a multi-tensor correlation coefficient showing better alignment of WM structures and improved abnormality detection compared to DT registration. However, their algorithm is centered on multi-tensors, while many other diffusion models are available [10]. Furthermore, their measure requires the one-to-one mapping of the compared MCMs tensors. This task is time-consuming and may lead to discontinuities of the similarity measure and its derivative as illustrated in Section 3.2. This mapping is also harder to define when models with different number of compartments are compared, as it happens if model selection [11] is part of MCM estimation. As an alternative, Cheng et al. [4] proposed to use the $\ell_{2}$ norm ; their approach is however limited to multi-tensors and to a sum of squared differences (SSD).

We propose in Section 2 new similarity measures for MCM images registration, considering distances between MCM characteristic functions $(\mathrm{CF})$, i.e. the Fourier transforms of the MCM probability density functions (PDF). The first measure extends the SSD to MCM ; the second measure proposes a surrogate on MCM to the scalar correlation coefficient (CC) to account for variability across patients and acquisition parameters. Both measures are agnostic to individual compartment types, i.e. we assume that their CF describe the microstructure well for registration, and only require to derive the generic expression of the inner product of two compartments to get an analytical form. For complex models, we propose a discrete spherical approximation to both measures. We evaluate the proposed measures in Section 3 using simulated and in-vivo data showing their smoother evolution and improved performance for matching WM structures.

\section{Methods}

\subsection{Diffusion Multi-Compartment Models}

We define a diffusion MCM $M$ as a weighted sum of $N$ compartments, each depicting water diffusion in a specific environment. For example, an MCM may contain an isotropic free water compartment and several directional compartments, each describing diffusion in a fiber bundle with a given orientation. The MCM admits a PDF $p_{M}(x)=\sum_{i=1}^{N} w_{M, i} p_{M, i}(x)$, where $p_{M, i}$ is the PDF of the $i$-th compartment $\left(M_{i}\right)$ of the model and $w_{M, i}$ is the weight of compartment $M_{i}\left(\sum_{i} w_{M, i}=1\right) \cdot p_{M, i}$ can take one of many different forms [10]. $M$ also admits a unique $\operatorname{CF} \varphi_{M}(t)=\sum_{i=1}^{N} w_{M, i} \varphi_{M, i}(t)$, where $\varphi_{M, i}$ is the CF of $M_{i}$. As compartments are usually antipodally symmetric, $\varphi_{M}$ takes its values in $\mathbb{R}$.

\section{$2.2 \ell_{2}$ Space of Square Integrable Functions}

We consider the Hilbert space $\mathcal{L}$ of real-valued square integrable functions $f$ such that $f: \mathbb{R}^{3} \rightarrow \mathbb{R}$ and $\int_{\mathbb{R}^{3}} f^{2}(x) d x<\infty$. The inner product on $\mathcal{L}$ is de- 
fined as $\langle f, g\rangle_{\mathcal{L}}=\int_{\mathbb{R}^{3}} f(x) g(x) d x$ and is associated to a distance $d_{\mathcal{L}} \cdot d_{\mathcal{L}}$ can be used to compare MCM PDFs since most fascicle model PDFs are square integrable. However we define our similarity measures on MCM CFs, i.e. the Fourier transforms of the PDFs, since: 1- the CF is usually simpler than the PDF and is directly related to DWI signal formation, 2- distances on CFs or PDFs are equivalent since $d_{\mathcal{L}}$ computed on CFs or PDFs are equal (Plancherel theorem). The distance between two MCM CFs $\varphi_{X}$ and $\varphi_{Y}$ is written as follows:

$$
d_{\mathcal{L}}^{2}\left(\varphi_{X}, \varphi_{Y}\right)=\mathbf{w}_{\mathbf{X}}{ }^{T} \mathbf{A}_{\mathbf{X}, \mathbf{X}} \mathbf{w}_{\mathbf{X}}+\mathbf{w}_{\mathbf{Y}}^{T} \mathbf{A}_{\mathbf{Y}, \mathbf{Y}} \mathbf{w}_{\mathbf{Y}}-2 \mathbf{w}_{\mathbf{X}}{ }^{T} \mathbf{A}_{\mathbf{X}, \mathbf{Y}} \mathbf{w}_{\mathbf{Y}}
$$

where $\mathbf{w}_{\mathbf{X}}$, respectively $\mathbf{w}_{\mathbf{Y}}$, represents the vector of compartment weights for model $X$, respectively for model $Y$. $\mathbf{A}_{\mathbf{X}, \mathbf{Y}}$ denotes the $N_{X} \times N_{Y}$ matrix of all pairwise inner products between the individual compartments of $X$ and $Y$. As a consequence, $d_{\mathcal{L}}$ can be computed analytically if $\langle.,\rangle_{\mathcal{L}}$ can be.

\subsection{Similarity Measures}

We consider measures computed over a set of voxels that have been paired between two images, respectively $\mathbf{R}=\left\{R_{1}, \cdots, R_{N}\right\}$ and $\mathbf{F}=\left\{F_{1}, \cdots, F_{N}\right\}$, where $R_{k}$ and $F_{k}$ denote the $k$-th paired MCMs.

MCM Sum of Squared Differences The first measure is the direct extension of SSD to MCMs relying on the direct comparison of the MCM paired between $\mathbf{R}$ and $\mathbf{F}$ using the distance defined in Eq. (1):

$$
\operatorname{SSD}_{\mathcal{L}}(\mathbf{R}, \mathbf{F})=\sum_{k=1}^{N} d_{\mathcal{L}}^{2}\left(\varphi_{R_{k}}, \varphi_{F_{k}}\right)
$$

where $\varphi_{R_{k}}$, respectively $\varphi_{F_{k}}$ is the CF of the $k$-th MCM in $\mathbf{R}$, respectively in F. Assuming all compartments are tensors, this measure admits a closed-form solution based on an inner product of the form: $\left\langle\varphi_{i}, \varphi_{j}\right\rangle_{\mathcal{L}}=(2 \pi)^{3 / 2}\left|\Sigma_{i}+\Sigma_{j}\right|^{-1 / 2}$.

MCM Correlation Surrogate Similarly to the scalar $\mathrm{SSD}, \mathrm{SSD}_{\mathcal{L}}$ may suffer from changes due to inter-patient brain variability or DWI acquisition differences. We therefore present a second measure aimed at being an "equivalent", in the $\mathcal{L}$ space, to the scalar squared CC. Going back to the scalar case, the squared CC can be seen as a measure of the linear relationship between two sets of scalars. Another way of measuring this relationship has been proposed by Alpert et al. [2] as the squared residual of the linear regression between these sets. We propose an MCM correlation surrogate in $\mathcal{L}$ following a similar idea:

$$
C_{\mathcal{L}}=\min _{\boldsymbol{\theta}} \sum_{k=1}^{N} d_{\mathcal{L}}^{2}\left(\varphi_{R_{k}}, H_{\boldsymbol{\theta}} \varphi_{F_{k}}\right)
$$


where $H_{\boldsymbol{\theta}}: \mathbb{R}^{3} \rightarrow \mathbb{R}$ denotes a kernel function with parameters $\boldsymbol{\theta}$, such that it preserves the square integrability of $\varphi_{F_{k}}$. $C_{\mathcal{L}}$ is the residual of the minimization of Eq. (3) with respect to $\boldsymbol{\theta}$. Interestingly, if $H_{\boldsymbol{\theta}}$ is a CF, Eq. (3) is equivalent to the convolution of $p_{F_{k}}$ with $H_{\boldsymbol{\theta}}$ PDF. Following this, we choose $H_{\boldsymbol{\theta}}$ as the CF of the centered Gaussian PDF with parameter $\sigma: H_{\boldsymbol{\theta}}(t)=\exp \left(-\sigma t^{T} t / 2\right)$, which results in a Gaussian smoothing of the MCMs PDF.

Developing further Eq. (3) leads to the computation of three different inner products of the form: $\left\langle\varphi_{i}, H_{\boldsymbol{\theta}}^{\alpha} \varphi_{j}\right\rangle_{\mathcal{L}}$ for $\alpha \in\{0,1,2\}$. Similarly to $\operatorname{SSD}_{\mathcal{L}}$, when considering tensor MCM compartments, these inner products admit an analytical form $\left\langle\varphi_{i}, H_{\theta}^{\alpha} \varphi_{j}\right\rangle_{\mathcal{L}}=(2 \pi)^{3 / 2}\left|\Sigma_{i}+\Sigma_{j}+\alpha \sigma I d\right|^{-1 / 2}$ (where $I d$ denotes the $3 \times 3$ identity matrix). In this specific case, optimization over $\boldsymbol{\theta}$ is carried out using gradient-based optimization algorithm as analytical derivatives are available.

Similarity Metrics Spherical Approximation For some non Gaussian compartment types, the inner products cannot be computed analytically. We therefore propose a discrete approximation $\langle., .\rangle_{a \mathcal{L}}$ to the inner product $\langle.,,\rangle_{\mathcal{L}}$ using the fact that CFs decrease fast to infinity and are linked to DWI signal formation as: $S_{k}=S_{0} \varphi\left(\sqrt{2 b_{k}} g_{k}\right)$ where $S_{0}$ is the nominal signal without diffusion weighting and $S_{k}$ the diffusion weighted signal with b-value $b_{k}$ along gradient $g_{k}$. CFs are used to estimate MCM parameters through a least squares minimization. We construct $\left\langle\varphi_{i}, \varphi_{j}\right\rangle_{a \mathcal{L}}$ by sampling values of $\varphi_{i}$ and $\varphi_{j}$ on DWI acquisition spheres, on a reasonable number of points (in our experiments we sampled a total of 169 gradients on 5 spheres ranging from $b=500$ to $3000 \mathrm{~s} . \mathrm{mm}^{-2}$ ):

$$
\left\langle\varphi_{i}, \varphi_{j}\right\rangle_{a \mathcal{L}}=\sum_{k} \Delta_{k} \varphi_{i}\left(\sqrt{2 b_{k}} g_{k}\right) \varphi_{j}\left(\sqrt{2 b_{k}} g_{k}\right)
$$

where $\Delta_{k}$ is the volume of a subpart of a sphere around point $\sqrt{2 b_{k}} g_{k}$. Plugging this approximation in Eqs. (2) and (3) defines two measures: $\mathrm{SSD}_{a \mathcal{L}}$ and $C_{a \mathcal{L}}$.

\subsection{Pairing-based MCM Similarity Measure}

As a comparison point to the proposed measures, we also recall the definition of a pairing-based SSD, denoted $\mathrm{SSD}_{P}$. The distance between two MCM $R_{k}$ and $F_{k}$ is defined through pairings between the MCM compartments $R_{k, i}$ and $F_{k, j}$. Then, assuming that $N_{R_{k}}=N_{F_{k}}$, the pairing-based distance $d_{P}$ is defined as:

$$
d_{P}^{2}\left(R_{k}, F_{k}\right)=\min _{a_{1} \ldots a_{N_{R_{k}}}} \sum_{i=1}^{N_{R_{k}}} w_{R_{k, i}} w_{F_{k, a_{i}}} d_{I}^{2}\left(p_{R_{k, i}}, p_{F_{k, a_{i}}}\right)
$$

where $a_{i}$ are elements of an association vector a mapping each element of $R_{k}$ to an element of $F_{k}, d_{I}$ is a distance between MCM compartment PDFs: for multi-tensors the log-Euclidean distance [3]. As a is unknown, all permutations are tested and $d_{P}$ is the minimum over those associations. The sum of $d_{P}^{2}$ over $k$ defines $\mathrm{SSD}_{P}$. An additional difficulty appears when $N_{R_{k}}<N_{F_{k}}$ when model selection was performed, making a more complicated to define. We chose to pair each element of $R_{k}$ to a single element of $F_{k}$, extraneous elements being left out. 


\section{Experiments and Results}

\subsection{Image Database and Registration Algorithm}

We have used in vivo data from 10 pre-processed healthy subjects from the Human Connectome Project $(\mathrm{HCP})^{1}$. Each DWI acquisition includes 270 gradient directions on three different b-values (from $b=1000$ to $3000 \mathrm{s.mm} \mathrm{mm}^{-2}$ ). The image size is $145 \times 174 \times 145$ and voxel size $1.25 \times 1.25 \times 1.25 \mathrm{~mm}^{3}$. From the DWI, multi-tensor models were estimated so that the approximate and analytical measures can be compared. Estimation was performed using Scherrer et al. method with model selection [11]. Each multi-tensor model was composed of 2 isotropic compartments (free water and restricted water) and a maximum of 3 tensors.

As a fair evaluation framework, we have integrated all measures $\operatorname{SSD}_{P}$, $\left.\mathrm{SSD}_{\mathcal{L}}, \mathrm{SSD}_{a \mathcal{L}}, C_{\mathcal{L}}, C_{a \mathcal{L}}\right)$ in a generic MCM non linear registration algorithm. This algorithm is an extension of a block-matching scalar image registration [5]. Two essential components were specified for MCM registration. First interpolation for image resampling was performed using state-of-the-art MCM interpolation techniques [12]. The second major brick is the re-orientation technique for resampling. We used the preservation of principal direction technique [1] on each compartment of the interpolated MCM. We used this framework in our experiments with the same parameters, changing only the similarity measure.

\subsection{Similarity Measures Qualitative Evaluation}

We evaluated the behavior of each similarity measure when translating or rotating a block (close to the ventricles, see Fig. 1.a) where the number of compartments is known to change. The selected block had a resolution of $5 \times 5 \times 5$ voxels and was either translated along the $\mathrm{Y}$-axis (vertical axis in Fig. 1.a) or rotated around the Z-axis (transverse axis in Fig. 1.a). For each case, we computed the similarity measure between the transformed block and the original one. We report in Fig. 1 the five evaluated similarity measures, as a function of the transformation parameters, scaled so that their maximum is equal.

When rotating the block around its center (Fig. 1.b), all measures perform well and indicate one clear global minimum although $\mathrm{SSD}_{P}$ suffers from some irregularities. The main variations appear in the translation case due to the change of explored region with the increasing translation. In Fig. 1.c, $\mathrm{SSD}_{P}$ appears more sensitive to interpolation than the proposed measures. The value of $\mathrm{SSD}_{P}$ may vary abruptly when the MCM compartment pairings change, leading to non differentiability. The mapping function a also maps one compartment to one and only one other compartment. However, the number of compartments is different when translation occurs. Some compartments are thus left out, leading to part of one MCM not being considered in the measure.

Measures based on the $\mathcal{L}$ space compare MCMs as a whole and therefore adopt much smoother and regular shapes. Moreover, the shapes of the approximations are very similar (the green and blue curves are overlapping in Fig. 1 as

\footnotetext{
${ }^{1}$ http://www.humanconnectome.org
} 


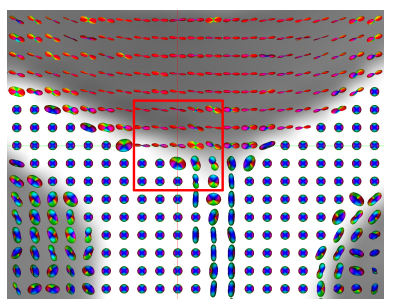

(a) Block position

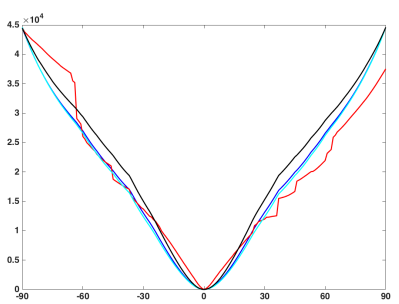

(b) $\mathrm{Z}$ axis rotation (degrees)

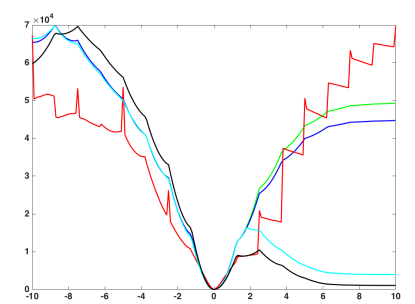

(c) $\mathrm{Y}$ axis translation $(\mathrm{mm})$

Fig. 1. Similarity measures evolution around the corpus callosum for (b): rotation and (c): translation. Legend: red: $\mathrm{SSD}_{P}$, green: $\operatorname{SSD}_{\mathcal{L}}$, blue: $\operatorname{SSD}_{a \mathcal{L}}$, cyan: $C_{\mathcal{L}}$, black: $C_{a \mathcal{L}}$.

well as the cyan and black curves in Fig. 1.b), suggesting the discrete approximation is well fit for MCM registration. $C_{\mathcal{L}}$ and $C_{a \mathcal{L}}$ adopt a different shape from the SSD measures, allowing for more possibilities in matching one block with other regions while keeping the true matching as the global optimum.

\subsection{Quantitative Evaluation on HCP Data}

Simulated Deformations We simulated deformations by generating for each HCP subject a set of 5 poly-rigid transformations [5] by randomly seeding 15 local rigid transformations (rotations between $[-\pi / 6 ; \pi / 6]$ radians around each axis and translations between $[-10 ; 10] \mathrm{mm}$ in each direction) inside the brain and extrapolating a dense deformation. These transformations were then applied to their corresponding subject, generating two images to register: $I \circ T^{-1 / 2}$ and $I \circ T^{1 / 2}$. The ground truth transformation is then $T$. We present in Fig. 2 the voxelwise transformation errors for the registration with each measure.

All $\mathcal{L}$ space measures outperform $\mathrm{SSD}_{P}$. This is particularly visible around the ventricles (arrows on Fig. 2). This confirms previous results where $\operatorname{SSD}_{P}$ shape was irregular and thus more difficult to optimize in this region. The results obtained by the spherical approximations of the measures are close to their analytical counterparts. All $\mathcal{L}$ space measures appear to perform equally well, only displaying subtle error differences. We also computed the average transformation error over the whole image and averaged those across all experiments. All measures improve over the initial error (2.704). The $\operatorname{SSD}_{P}$ error $(0.587)$ is however significantly higher (paired t-test, $p<0.05$ ) than the $\mathcal{L}$ space measures: $\mathrm{SSD}_{\mathcal{L}}, \mathrm{SSD}_{a \mathcal{L}}$ and $C_{\mathcal{L}}$ reach an average error of 0.577 and $C_{a \mathcal{L}}$ reaches 0.578 . All proposed measures are not significantly different from each other (paired t-test, $p>0.05$ ). The absence of difference between MCM correlation and SSD may be explained by the fact that each couple of images comes from the same subject and $\mathrm{SSD}_{\mathcal{L}}$ is enough in that case to recover the deformation.

Inter-subject registration We quantitatively evaluated inter-subject registration by registering all couples of HCP images (total of 90 registrations) and applying the deformation to WM segmentations. These parcellations were obtained 


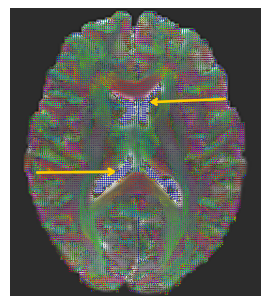

(a) Reference image

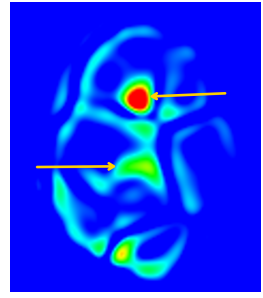

(d) $\mathrm{SSD}_{P}$

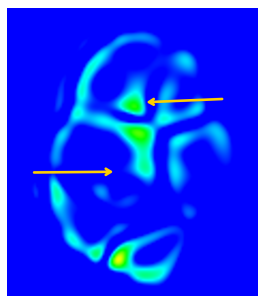

(b) $\operatorname{SSD}_{a \mathcal{L}}$

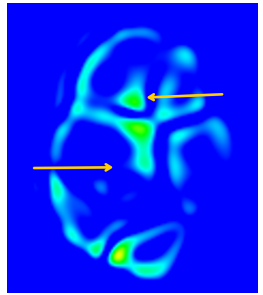

(e) $\operatorname{SSD}_{\mathcal{L}}$

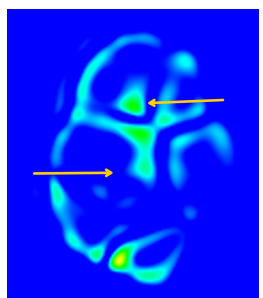

(c) $C_{a \mathcal{L}}$

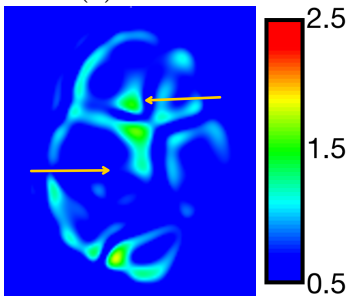

(f) $C_{\mathcal{L}}$

Fig. 2. Transformation errors after registration on a representative HCP subject: fixed MCM image (a), error using $\operatorname{SSD}_{P}(\mathrm{~d}), \operatorname{SSD}_{\mathcal{L}}(\mathrm{e}), \operatorname{SSD}_{a \mathcal{L}}$ (b), $C_{\mathcal{L}}$ (f), $C_{a \mathcal{L}}$ (c).

by the HCP pipelines using FreeSurfer and contain a total of 182 structures. We report in Fig. 3 the averaged total overlaps (TO) [9] between the transformed parcellations and the true ones for each method.

The average TO are relatively low for all methods. This comes from intersubject variability of the reference parcellations. The overlap scores however confirm previous findings: the $\mathcal{L}$ space measures all outperform $\mathrm{SSD}_{P}$ (average TO: $47.23 \%$ ), itself being better than global affine registration on B0 images (all results significant: paired t-test, $p<0.05$ ). This confirms that the pairing based measure, although good overall, encounters more difficulties. $C_{\mathcal{L}}$ performs the best over all other measures (average TO: $50.73 \%$, paired t-test, $p<0.05$ ), while both $\mathrm{SSD}_{\mathcal{L}}$ and $\mathrm{SSD}_{a \mathcal{L}}$ perform equally well (average TO: $50.53 \%$ ). Introducing a more flexible similarity measure than SSD may thus help MCM image registration. $C_{a \mathcal{L}}$ performs a little lower than other $\mathcal{L}$ space measures (average TO:

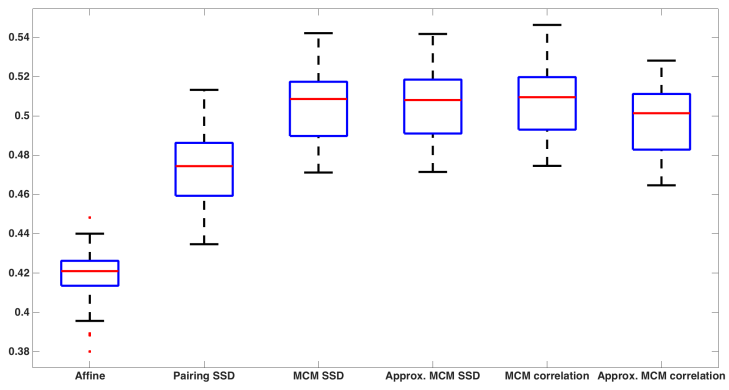

Fig. 3. White matter parcellation total overlaps after registration of MCM images from HCP data, using either global affine registration, or non linear registration with respectively $\mathrm{SSD}_{P}$, $\mathrm{SSD}_{\mathcal{L}}, \mathrm{SSD}_{a \mathcal{L}}, \mathrm{MCM}$ correlation surrogate $C_{\mathcal{L}}$ and $C_{a \mathcal{L}}$. 
$49.73 \%$ ), especially compared to $C_{\mathcal{L}}$. This suggests that the selected approximation points may have an influence on the approximation quality.

\section{Conclusion}

We have proposed a new framework to define MCM similarity measures based on the fact that most MCM CF belong to the Hilbert space of square integrable functions $\mathcal{L}$. Defining distances between MCM in $\mathcal{L}$ has two main advantages: 1- no pairing is needed between the individual MCM compartments thereby alleviating the complex computation of a mapping function; 2- the measures are generic as they are agnostic to the compartments models. We defined two similarity measures (and their analytical expressions for multi-tensors): an MCM SSD and an MCM surrogate to the scalar CC. In addition, we defined a numerical approximation applicable to all $\mathcal{L}$ space based measures for complex models. We have evaluated these new similarity measures on both simulated transformations and real data from HCP, showing the better behavior of $\mathcal{L}$ space measures and the potential of the MCM correlation surrogate.

\section{References}

1. Alexander, D., et al.: Spatial transformations of diffusion tensor magnetic resonance images. IEEE TMI 20(11), 1131-1139 (2001)

2. Alpert, N., Berdichevsky, D., Levin, Z., Morris, E., Fischman, A.: Improved methods for image registration. Neuroimage 3, 10-18 (1996)

3. Arsigny, V., Fillard, P., Pennec, X., Ayache, N.: Log-Euclidean metrics for fast and simple calculus on diffusion tensors. MRM 56(2), 411-421 (2006)

4. Cheng, G., Vemuri, B.C., et al.: Non-rigid registration of hardi represented by Gaussian mixture fields. In: MICCAI. LNCS, vol. 5761, pp. 190-197 (2009)

5. Commowick, O., et al.: Automated diffeomorphic registration of anatomical structures with rigid parts: application to dynamic cervical MRI. In: MICCAI. LNCS, vol. 7511 , pp. 163-170 (2012)

6. Dhollander, T., et al.: Spatial transformations of high angular resolution diffusion imaging data in Q-space. In: MICCAI CDMRI workshop. pp. 73-83 (2010)

7. Goodlett, C., et al.: Group analysis of DTI fiber tract statistics with application to neurodevelopment. Neuroimage 45(1(S1)), S133-S142 (2009)

8. Jensen, H., Lauze, F., Nielsen, M., Darkner, S.: Locally orderless registration for diffusion weighted images. In: MICCAI. LNCS, vol. 9350, pp. 305-312 (2015)

9. Klein, A., et al.: Evaluation of 14 nonlinear deformation algorithms applied to human brain MRI registration. NeuroImage 46(3), 786-802 (July 2009)

10. Panagiotaki, E., et al.: Compartment models of the diffusion MR signal in brain white matter: A taxonomy and comparison. Neuroimage 59(3), 2241-2254 (2012)

11. Scherrer, B., Warfield, S.: Parametric representation of multiple white matter fascicles from cube and sphere diffusion MRI. PLoS One 7(11), e48232 (2012)

12. Taquet, M., Scherrer, B., et al.: A mathematical framework for the registration and analysis of multi-fascicle models for population studies of the brain microstructure. IEEE TMI 33(2), 504-517 (2014)

13. Yeo, B.T.T., Vercauteren, T., et al.: DT-REFinD: Diffusion tensor registration with exact finite-strain differential. IEEE TMI 28(12), 1914-1928 (2009) 